\title{
Shift from Hippocampal to Neocortical Centered Retrieval Network with Consolidation
}

\author{
Atsuko Takashima, ${ }^{1}$ Ingrid L.C. Nieuwenhuis, ${ }^{1}$ Ole Jensen, ${ }^{1}$ Lucia M. Talamini, ${ }^{3}$ Mark Rijpkema, ${ }^{1}$ \\ and Guillén Fernández ${ }^{1,2}$ \\ ${ }^{1}$ Donders Institute for Brain, Cognition, and Behaviour, Radboud University Nijmegen and ${ }^{2}$ Department of Neurology, Radboud University Nijmegen \\ Medical Centre, 6500 HB, Nijmegen, The Netherlands, and '3Department of Psychonomics, University of Amsterdam, 1018 WB Amsterdam, \\ The Netherlands
}

The standard model of system-level consolidation posits that the hippocampus is part of a retrieval network for recent memories. According to this theory, the memories are gradually transferred to neocortical circuits with consolidation, where the connections within this circuit grow stronger and reorganized so that redundant and/or contextual details may be lost. Thus, remote memories are based on neocortical networks and can be retrieved independently of the hippocampus. To test this model, we measured regional brain activity and connectivity during retrieval with functional magnetic resonance imaging. Subjects were trained on two sets of face-location association and were tested with two different delays, $15 \mathrm{~min}$ and $24 \mathrm{~h}$ including a whole night of sleep. We hypothesized that memory traces of the locations associated with specific faces will be linked through the hippocampus for the retrieval of recently learned association, but with consolidation, the activity and the functional connectivity between the neocortical areas will increase. We show that posterior hippocampal activity related to high-confidence retrieval decreased and neocortical activity increased with consolidation. Moreover, the connectivity between the hippocampus and the neocortical regions decreased and in turn, cortico-cortical connectivity between the representational areas increased. The results provide mechanistic support for a two-level process of the declarative memory system, involving initial representation of new associations in a network including the hippocampus and subsequent consolidation into a predominantly neocortical network.

\section{Introduction}

Medial temporal lobe lesions cause temporally graded retrograde amnesia, suggesting a time-limited role of the hippocampus in memory retrieval (Scoville and Milner, 1957; Squire and ZolaMorgan, 1991) [but see Nadel and Moscovitch (1997) and Moscovitch et al. (2006)]. This phenomenon forms the basis of the standard model of system-level consolidation, which proposes that the hippocampus is part of a retrieval network for recent memories, but that memories are gradually transferred to neocortical circuits for long-term storage (Alvarez and Squire, 1994; Smith and Squire, 2009). Animal studies have confirmed that after time periods of a few weeks, lesioning the hippocampus no longer affects successful retrieval (Zola-Morgan and Squire, 1990; Tse et al., 2007). In contrast, neocortical contributions to memory retrieval increase (Bontempi et al., 1999; TakeharaNishiuchi and McNaughton, 2008). Also in humans, hippocampal activity related to successful item recognition decreases over

\footnotetext{
Received Feb. 13, 2009; revised July 2, 2009; accepted July 7, 2009.

This research was supported by grants from the Netherlands Organization for Scientific Research 051.04 .100 918.66.613, and 451-06-006). We thank Paul Gaalman and Bram Daams for technical support, Lennart Verhagen for data analyses, and Marijn Kroes for constructive comments on the manuscript.

Correspondence should be addressed to Atsuko Takashima, Donders Institute for Brain, Cognition, and Behaviour, Radboud University Nijmegen, P.0. Box 9101, 6500 HB, Nijmegen, The Netherlands. E-mail: atsuko.takashima@donders.ru.nl.

DOI:10.1523/JNEUROSCI.0799-09.2009

Copyright $\odot 2009$ Society for Neuroscience $\quad$ 0270-6474/09/2910087-07\$15.00/0
}

time (Takashima et al., 2006) [but see, e.g., Bosshardt et al. (2005a) and Janzen et al. (2008)].

Computational models provide a rationale for system-level consolidation (McClelland et al., 1995; O’Reilly and Norman, 2002). The hippocampus is suited to encode information rapidly online, but this rapid plasticity makes representations vulnerable to overwriting. On the other hand, the neocortex has a slower learning rate, allowing integration of new information into existing knowledge in a more efficient way by coding nonoverlapping, generalized, and structured information (Marr, 1970, 1971). Derived from lesion studies and computational modeling, the system-level consolidation theory proposes that different event features, represented in distributed neocortical areas, are initially linked together by the hippocampus. With consolidation, information is integrated into neocortical circuits and reorganized in the process so that it can ultimately be retrieved independently of the hippocampus. Putatively, this integration and reorganization could entail reduction of noncentral and overlapping contextual details.

Experimental findings support this notion by showing that hippocampal contributions to memory retrieval decrease over time, but proposed changes in connectivity with consolidation have rarely been investigated. Although there are studies assessing differences in connectivity between groups of subjects (Addis et al., 2007; Gais et al., 2007; Sterpenich et al., 2007), only one study in cats reports changes in functional connectivity with consolidation (Paz et al., 2007). Lesion studies cannot readily show 
changes in interaction between different regions. Recent functional magnetic resonance imaging (fMRI) techniques provide a unique opportunity to probe such changes (Levine et al., 2004; Addis and McAndrews, 2006).

Here we report a study probing consolidation-related changes in brain activity associated with retrieval of facelocation associations. In addition to local activity assessment for the two different retrieval time points, we used functional connectivity analysis (Friston et al., 1997) to reveal changes in network properties. In line with the system-level consolidation theory, we hypothesized that highconfidence retrieval of recently learned face-location associations would involve hippocampal activity and a strong functional connectivity between the hippocampus and representational areas in the neocortex. With consolidation, this network is expected to change, such that functional connectivity between the neocortical areas increases, while hippocampal activity and its connectivity to these representational areas decrease.

\section{Materials and Methods}

Subjects. Twenty-seven subjects were recruited from the university campus in Nijmegen, The Netherlands ( 8 males, age mean \pm SD: $24.5 \pm$ 4.6 years). All subjects had normal or corrected-to-normal vision. Subjects received a monetary payment for their participation. Written informed consent was obtained according to the local medical ethics committee. A sleep questionnaire was obtained and all subjects reported to be regular night-time sleepers.

Stimuli. One hundred twenty grayscale front-facing photographs of unfamiliar male and female faces (60 of each) were used as face stimuli. These pictures were taken from anonymous visitors of a museum in Amsterdam with a digital camera. The purpose of the photos was explained to the visitors and they gave consent to the usage of their face portraits as experimental stimuli. The pictures comprised the neck and above, all showing neutral expressions gazing toward the camera. These faces were divided into two sets of 60 faces each ( 30 male and 30 female). One set of faces was encoded on day 1 and assigned to the remote condition and the other was encoded on day 2 and assigned to the recent condition (Fig. 1A). The two stimulus sets were randomly assigned to either remote or recent conditions for each subject.

Procedure. For the remote condition, subjects learned the first set of 60 face-location associations on day 1 . Before the actual memorization of the association, they performed a face item familiarization task, to minimize effects of item memory. Subjects were exposed to 60 faces over three cycles while they responded to simple questions about the face (How old do you think he/she is? Do you like this face? Is this face male or female?). After this familiarization task, subjects were trained on the face-location associations (learning session) (Fig. $1 A$ ). Each subject underwent three encoding-retrieval cycles. During encoding, subjects passively viewed each face while it was associated with one of the six locations on the screen. Immediately after each encoding block, a retrieval block followed in which the subjects were instructed to indicate the correct location of each face by a joystick movement toward the previously learned position with a confidence rating (Fig. $1 B$ ). No feedback was given. This encoding-retrieval cycle was repeated three times with the same set of faces associated with identical locations, but in different random presentation orders. Each location was used equally often across the stimuli. For the recent condition, subjects performed the same familiarization task and learning session $24 \mathrm{~h}$ later (day 2) as on day 1, but with a new set of 60 faces.

Approximately $15 \mathrm{~min}$ after completing the learning session (recent set) on day 2, subjects performed a cued recall memory test for facelocation memory during MRI data acquisition. All faces of the remote and recent conditions served as cues, and were presented centrally one at a time, in randomized order. Subjects were instructed to indicate the correct location by a joystick movement using their right hand. The face and the gray location probes stayed on screen for $4 \mathrm{~s}$ (Fig. $1 B$ ). If the subjects responded to the correct location within this $4 \mathrm{~s}$, the trial was considered correct. No feedback was given. After a jittered interval of 1-2 $\mathrm{s}$, subjects were additionally instructed to rate the confidence of their response $(1=$ unsure to $5=$ sure $)$. In case they did not remember the location, they were instructed to indicate the lowest confidence rating of 1. Each trial was followed by a jittered intertrial interval of 3-7 s during which a fixation cross was presented in the center of the screen. Twentyfive null events (fixation cross) with duration of $16 \mathrm{~s}$ each were randomly intermixed between trials.

To avoid that task execution was new to the subjects during the first learning session but familiar during the second learning session, subjects underwent an identical learning session as described above with stimuli not tested in the final cued-recall, $\sim 1$ week before the actual experiment.

MRI data acquisition. For fMRI, we acquired $\mathrm{T} 2^{*}$-weighted images covering the whole brain using an echo-planar imaging (EPI) sequence (31 axial slices, ascending slice acquisition, repetition time $(\mathrm{TR})=2.28 \mathrm{~s}$, echo time $(\mathrm{TE})=35 \mathrm{~ms}, 90^{\circ}$ flip angle, matrix $=64 \times 64$, slice thickness: $3.5 \mathrm{~mm}$, slice gap: $0.35 \mathrm{~mm}$, field of view (FOV): $224 \mathrm{~mm}$, Sonata $1.5 \mathrm{~T}$, Siemens). For structural MRI, we acquired T1-weighted images using a magnetization-prepared, rapid-acquisition gradient echo sequence (176 sagittal slices, $\mathrm{TR}=2250 \mathrm{~ms}, \mathrm{TE}=2.95 \mathrm{~ms}, 15^{\circ}$ flip angle, matrix $=$ $256 \times 256$, slice thickness: $1.0 \mathrm{~mm}$, FOV: $256 \mathrm{~mm}$ ).

MRI data analysis. Image preprocessing and statistical analysis was performed using SPM5 (www.fil.ion.ucl.ac.uk). The first five volumes of each participant's functional EPI data were discarded to allow for T1 
equilibration. The EPI images were realigned and the subject mean was coregistered with the corresponding structural MRI using mutual information optimization. These EPI images were subsequently slice-time corrected to the 16th slice, using SPM5's Fourier panes shift interpolation. Both functional and structural scans were spatially normalized and transformed into a common Montreal Neurological Institute space (resampled at voxel size $2 \times 2 \times 2 \mathrm{~mm}$ ), as defined by the SPM5 T1.nii template, as well as spatially filtered by convolving the functional images with an isotropic three-dimensional (3D) Gaussian kernel (8 mm full width at half maximum). The normalized structural images were segmented into gray matter, white matter, and CSF area using unified segmentation algorithm implemented in SPM5 (Ashburner and Friston, 2005). These segmented images were used as masks to calculate mean intensity levels for white matter, CSF, and a residual compartment (section outside the brain and skull) for each of the functional images. The segmentation procedure was performed to create three regressors that describe non-BOLD signal intensity variance.

The fMRI data were analyzed statistically using the general linear model (GLM) and statistical parametric mapping. Five explanatory variables were included in the model: remote hits (associations learned on day 1 and responded correctly with a confidence rating of $5=$ sure), recent hits (associations learned on day 2 and responded correctly with a confidence rating of $5=$ sure), incorrect responses, null events, and trials of no interest comprising of all unsure correct trials from both remote and recent condition responded with confidence of 4 or below. These explanatory variables were temporally convolved with the canonical hemodynamic response function (HRF) along with its temporal derivatives provided by SPM5. Each event was time-locked to the presentation of the face. For the null events the whole 16 s block was modeled. The design matrix included the six head motion regressors (translations, rotations), their derivatives, and also three extra parameters consisting of mean intensity level in the white matter, CSF, and residual compartment (section outside the brain and skull) to account any residual movement- or signal intensity-related effect (Verhagen et al., 2006). A high pass filter was implemented using a cutoff period of $128 \mathrm{~s}$ to remove low-frequency effects from the time series. For statistical analysis, relevant contrast parameter images were generated for each subject and subsequently subjected to a second-level analysis (Penny et al., 2003) treating subjects as a random variable.

To probe changes in functional connectivity associated with consolidation, we performed a psychophysiological interaction (PPI) analysis (Friston et al., 1997) embedded in SPM5. In the PPI analysis, functional connectivity is assessed by searching for brain regions that coactivate with a seed region (physiological factor), and in which this coactivation is significantly different in one condition compared with the other (psychological factor). Here we analyzed areas that coactivated with the defined seed regions (explained in detail in the result section) more strongly for remote hits than recent hits and vice versa. A GLM was constructed at the single subject level using three regressors: (1) the deconvolved signal from the seed region, (2) trial onset for remote hits (positive) and recent hits (negative) convolved with HRF, and (3) the interaction term between the first and the second regressors, together with the 15 movement related regressors that were used in the GLM mentioned above. For each subject and for each seed region, the physiological activity was defined by the first eigenvariate extracted from the signals from the voxels within the defined seed region. Brain regions that showed a significant effect in the interaction contrast were considered to covary as a functional network with the seed region more strongly for the remote (or the recent) condition. This interaction contrast for every subject was used as input for the second-level random effects analysis.

Results of all second level analyses were initially thresholded at $p<$ 0.001 (voxel-level, uncorrected). For the whole-brain search, further cluster-size statistics were used as the test statistics applying a threshold of $p<0.05$ (corrected) (Hayasaka and Nichols, 2003).

\section{Results}

We used face-location associations for three reasons. First, the features of these stimuli lead to activity in clearly separated neocortical areas-faces are primarily represented in the fusiform gyrus, often referred to as fusiform face area (FFA) (Hirabayashi and Miyashita, 2005; Kanwisher and Yovel, 2006) and egocentric space is primarily represented in the posterior parietal cortex (PPC) (Levine et al., 1985; Takashima et al., 2007)—enabling us to examine changes in functional connectivity. Second, binding of between-domain association is known to involve the hippocampus (Mayes et al., 2007). Thirdly, by using a cued-recall paradigm, the retrieval of the associated information is known to activate the hippocampus (Diana et al., 2007; Mayes et al., 2007).

\section{Behavioral results}

Participants could have developed a better learning strategy when they encode the second (recent) set of face-location associations. To mitigate this potential order effect, participants performed a training session with stimuli not used in the actual experiment beforehand so that they had developed a learning strategy already for the first (remote) set. To test whether the two learning sessions led to similar performance levels by the end of the third cycle, correct rates and reaction times (RTs) for correct trials were compared between the final cycles of day 1 and day 2. Although RTs were slightly faster for the correct high-confidence recent trials $($ mean \pm SD: remote $=1.58 \pm 0.17 \mathrm{~s}$, recent $=1.51 \pm 0.17 \mathrm{~s}$ paired $t$ test $\left.t_{(26)}=2.08, p=0.05\right)$, correct rates were not significantly different between conditions (mean $\pm \mathrm{SD}$ : remote $=$ $89.4 \pm 10.5 \%$, recent $=91.4 \pm 11.0 \%$; paired $t$ test $t_{(26)}=1.19$, $p=0.24)$. Thus, the amount of associations that the subjects could retrieve at the end of the learning session was not significantly different.

During the test session inside the scanner, subjects were able to retrieve correct face-location associations well above chance level for both conditions (mean $\pm \mathrm{SD}$ correct rates: remote $=$ $65.9 \pm 12.8 \%$, recent $=89.9 \pm 11.8 \%$, chance level $=16.7 \%)$. As expected, recent associations were remembered better (correct rates: paired $t$ test $t_{(26)}=10.05, p<0.001$ ), faster (RTs for correct response: mean $\pm \mathrm{SD}$ : remote $=2.09 \pm 0.23 \mathrm{~s}$, recent $=1.67 \pm$ $0.20 \mathrm{~s}$; paired $t$ test $\left.t_{(26)}=11.68, p<0.001\right)$ and with higher confidence than remote ones (confidence rating: mean $\pm \mathrm{SD}$ : remote $=4.1 \pm 0.4$, recent $=4.7 \pm 0.3$; paired $t$ test $t_{(26)}=8.65$, $p<0.001)$. Although there was more forgetting in the remote condition, most subjects were able to remember enough associations with a very high confidence level of 5 (mean \pm SD number of trials out of 60 pairs: remote $=21.0 \pm 10.6$, recent $=45.9 \pm$ 11.6; RTs mean \pm SD: remote hits $=1.79 \pm 0.21 \mathrm{~s}$, recent hits $=$ $1.54 \pm 0.15 \mathrm{~s})$ for the analyses of the imaging data.

According to the self report, subjects slept on average $7.7 \pm$ $1.2 \mathrm{~h}$ between day 1 and day 2, which was similar to their usual nocturnal sleep length $(7.7 \pm 0.6 \mathrm{~h})$.

\section{Imaging}

We used only correct trials associated with the highest confidence rating (5) for both the remote and the recent condition (referred to hereafter as "remote hits" and "recent hits"). Hereby we controlled for a difference in recall certainties between remote and recent hits and thus mitigate activity reflecting differences in memory strength. Two subjects were removed from fMRI analyses, one due to excessive head movements and the other due to an insufficient number of trials (five remote hits and five recent hits). Thus, data of 25 subjects were used in the imaging analyses.

\section{Activity change with consolidation}

To reveal areas that increased their activity with consolidation, we contrasted brain activity of remote hits with recent hits. This contrast revealed a distributed set of activations, including the 
bilateral fusiform gyrus and the left PPC extending to the motor area (Fig. 2A; supplemental Table S1, available at www. jneurosci.org as supplemental material). The inferior frontal gyrus including Brodmann's areas 44 and 45 bilaterally, the precuneus bilaterally, and a cluster including the bilateral thalamus and basal ganglia also showed greater activity for remote hits.

To reveal those brain areas that decreased their activity with consolidation, we contrasted brain activity of recent hits with remote hits. In line with our hypothesis, the hippocampus bilaterally showed a decrease with consolidation (Fig. 2B). The cluster lies in the tail of the hippocampus (local maxima right: [18/-36/14] [18/-36/10], left: $[-28 /$ $-52 / 6] \quad[-30 /-42 / 2] \quad[-22 /-38 / 12])$. Since this part of the hippocampus runs along the wall of the posterior part of the lateral ventricle, the risk for motionrelated noise is increased. Therefore, we have added regressors of no interest in the GLM to account for any residual movement- or signal intensity-related effect that might cause false activity patterns in this area (see Materials and Methods for details). Thus, the reported cluster reflects changes in neural activation rather than motion-related noise. Even though the clusters overlaid onto the structural image may suggest activity in the lateral ventricles, the findings of functional connectivity analysis (reported in the next section) using this region supports our interpretation that these clusters do not reflect noise derived from the ventricular voxels. Furthermore, we tested the same functional connectivity analysis using a cluster with a similar size in the lateral ventricles close to the region of issue. There, we did not find any significant functional connectivity, which is also strong evidence that the active clusters are not reflecting ventricular effects.

To test whether the activity decrease or increase with consolidation was related to weakening of memory, we correlated behavioral performance with brain activity. We calculated the difference in the number of trials for recent hits and remote hits as a measure of forgetting. However, we did not find any significant effects.

\section{Functional connectivity change with consolidation}

To test whether functional connectivity of the posterior hippocampus decreased with consolidation, we took the above reported posterior hippocampal clusters as the seed region and conducted a PPI analysis embedded in SPM5 (Friston et al., 1997). In line with our hypotheses, the connectivity grew weaker with consolidation between the posterior hippocampus and the early visual areas bilaterally, extending to the FFA and the PPC bilaterally (Fig. 3A; supplemental Table S2, available at www. jneurosci.org as supplemental material). The reverse contrast (stronger connectivity with the posterior hippocampus after consolidation) did not show any significant clusters.

Since the task used faces as cues for the retrieval of associated locations, we did a second PPI analysis using the FFA as the seed region to investigate changes in connectivity with consolidation.
The seed region was defined as a $10 \mathrm{~mm}$ radial sphere on each side of the fusiform gyrus. The spheres were centered at the local maxima of the conjunction contrast of remote hits, recent hits, and incorrect conditions against the null events (left $[-36 /-68 /$ $-14]$; right $[34 /-52 /-12]$ ) — areas in the fusiform gyrus that were more active during observation of the face stimulus than the fixation cross. The seed region overlaps with the FFA reported earlier (Kanwisher and Yovel, 2006; Takashima et al., 2007). We observed both an increase (remote - recent) and a decrease (recent - remote) with consolidation in the connectivity with the FFA. The increase was observed between the FFA and early visual areas bilaterally extending to the fusiform gyrus, and the left PPC extending to the motor area (Fig. 3B, top; supplemental Table S3, available at www.jneurosci.org as supplemental material). A decrease with consolidation in connectivity was observed between the FFA seed region and the left anterior hippocampus, the left parahippocampal gyrus, and the right inferior to middle temporal lobe (Fig. 3B, bottom; supplemental Table S4, available at www.jneurosci.org as supplemental material). Two right middle hippocampal clusters (61 and 35 voxels) and a left posterior hippocampal cluster (74 voxels) were also observed (thresholded at voxel level $p<0.001$, uncorrected) but just failed to reach our conservative significance level at $p<0.05$, corrected (supplemental Fig. S2, available at www.jneurosci.org as supplemental material). With regard to the correlation with the behavioral performance difference, a positive correlation was found with a cluster in the left middle to inferior occipital cortex (local maximum $[-44 /-82 / 2])$ and a negative correlation was found in the right precuneus (local maximum $[22 /-58 / 28]$ ).

Thus the connectivity analyses show that the retrieval network for unconsolidated memory traces incorporates the hippocampus. However, after $24 \mathrm{~h}$, the network is more dependent on direct neocortical connections. 
A Functional connectivity with the posterior hippocampus

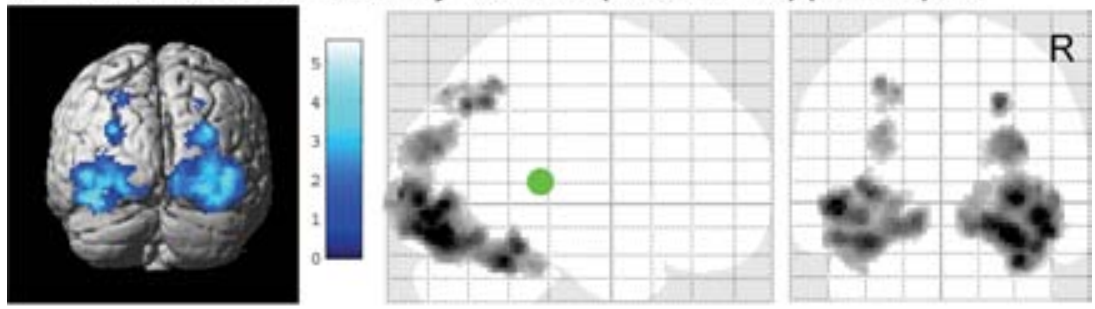

B Functional connectivity with the fusiform face area
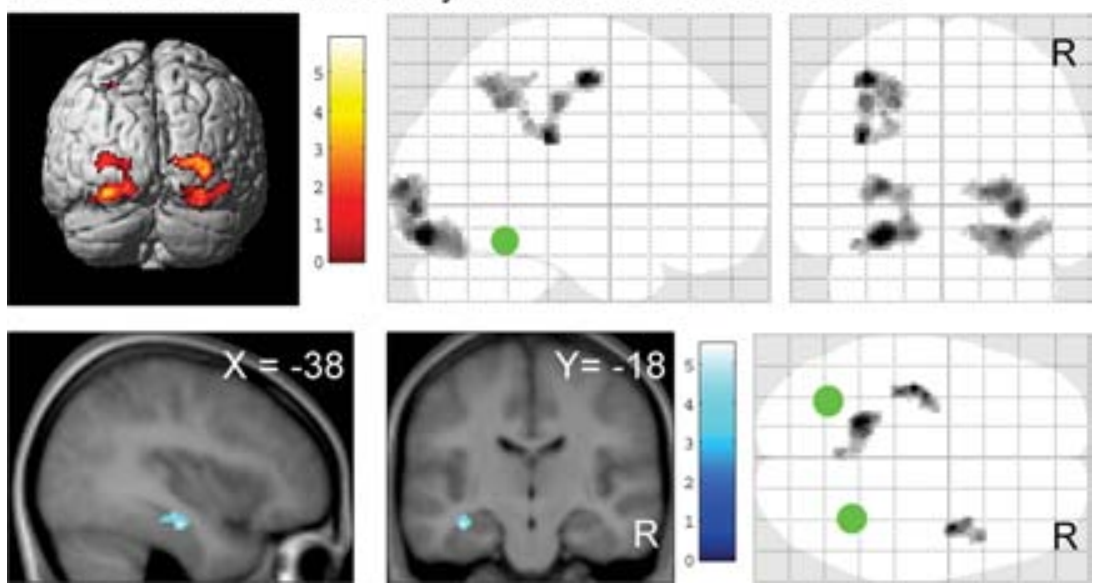

Figure 3. Networks that support retrieval of unconsolidated and consolidated memory traces. $\boldsymbol{A}$, Functional connectivity (PPI) with the posterior hippocampus. The connectivity to the early visual areas, extending to FFA, bilaterally and PPC bilaterally decreased with consolidation. $\boldsymbol{B}$, Functional connectivity with FFA. Increase with consolidation was observed to the early visual areas bilaterally and the left PPC extending to the motor cortex (top). Decrease with consolidation was observed in the left anterior hippocampus, the left parahippocampal gyrus and the right inferior to middle temporal lobe (bottom). $\boldsymbol{A}$ and $\boldsymbol{B}$ (top) show significant clusters superimposed onto a 3D image viewed from the back side and on intensity projection maps. $\boldsymbol{B}$ (bottom) shows the representative sagittal and coronal slices showing the significant cluster. Blue shows decrease with consolidation and red shows increase with consolidation. The circles in the sagittal and axial intensity projection maps represent the approximate location of the seed regions. Activations are depicted at $p<0.001$ uncorrected and cluster size that exceed 90 voxels for all panels, showing the significant clusters. R, Right.

\section{Discussion}

Here we provide initial experimental evidence in humans supporting systems-level consolidation theory. We find decreased hippocampal involvement in a retrieval network with ongoing consolidation, accompanied by an increase in cortico-cortical connectivity in specific representational areas.

Given the anatomical connectivity of the hippocampus to a wide range of areas in the brain (Suzuki and Eichenbaum, 2000; Muñoz and Insausti, 2005), it can be regarded as being in a highly favorable position to bind distributed neocortical nodes together during encoding and to index these nodes during retrieval. Moreover, due to high neuronal plasticity in the hippocampus, and as a consequence, even a single exposure to a stimulus changes its subsequent processing and enables later memory retrieval (Grunwald et al., 1998). Because of this rapid plasticity and optimal connectivity, the hippocampus is ideally suited for rapid encoding and retention of information by linking neocortical representations. However, over a lifetime, the hippocampus appears unlikely to be the long-term repository of all memories, and it seems probable that the process of consolidation involves transfer of memory traces to neocortical areas (Frankland and Bontempi, 2005). And above that, the accumulation of rote encoding is not suited for systematic and organized storing of information, even more so if the encoded element needs to be integrated into existing knowledge. According to the computational models, system-level consolidation involves fast hippocampal learning and slow neocortical learning which enables the memory trace to be encoded rapidly but also to be stored systematically, avoiding interference (Marr, 1970, 1971; McClelland et al., 1995). Here, we have shown that changes occurred from a hippocampus-dependent network to a less dependent network as a consequence of consolidation over a night of sleep. The retrieval of recent associations involved more activation in the posterior hippocampus than the retrieval of remote associations. In addition to that, we also show that functional connectivity between the posterior hippocampus and the memory representational areas, namely the FFA for faces and PPC for locations, was stronger for the recent than the remote associations. This is strong evidence for the notion that the hippocampus works as a linking node that binds neocortically distributed information for the retrieval of recently learned association (Addis et al., 2007; Diana et al., 2007). With consolidation, local activity in specific representational areas (FFA, PPC) increased and their mutual functional connectivity strengthened while connectivity between the FFA and the hippocampus weakened.

Although the critical role of the hippocampus in memory encoding and retrieval has unequivocally been shown, the issue of what happens with memories in the long-term is still under debate (Nadel and Moscovitch, 1997). The effect of hippocampal damage suggests a temporary role of the hippocampus in memory retrieval (Scoville and Milner, 1957; Zola-Morgan and Squire, 1990; Bontempi et al., 1999; Frankland and Bontempi, 2005). However, attempts to verify this notion in intact brains, using fMRI, have led to conflicting results. While some studies report decreased hippocampal activity with consolidation (Bosshardt et al., 2005b; Takashima et al., 2006), others show increases or no change (Addis et al., 2004; Gilboa et al., 2004; Bosshardt et al., 2005a; Piefke and Fink, 2005; Rekkas and Constable, 2005; Gais et al., 2007; Spiers and Maguire, 2007; Janzen et al., 2008). These studies have led to the interpretation that certain memories may never become independent of the hippocampus (Nadel and Moscovitch, 1997; Moscovitch et al., 2006). Retrieval of autobiographical memories is often reported to engage hippocampal activity (Gilboa et al., 2004) [for review, see Svoboda et al. (2006) and Cabeza and St Jacques (2007)]. However, testing for these memories by its remoteness might be problematic in the sense that these memories could have undergone reencoding due to memory retrieval between the actual occurrence of the event and test. We circumvented these issues by equating encoding by implementing a prospective, fully controlled procedure. Our results support the view that the hippocampal contribution to successful memory retrieval, decreases with consolidation. There are reports showing the presence of hippocampal activity after consolidation in studies using prospective paradigms such as word- 
pair retrieval or navigational memories. For instance, Bosshardt et al. (2005a) report an increase in the left midhippocampus with consolidation. We found similar activity for both recent and remote hits in the midhippocampal region. However, the activation strength was not different between the two conditions in our study. Bosshardt et al. (2005a) also report a right posterior hippocampal activity decrease, which is in line with our observation. In the review on studies investigating patients with hippocampal lesions, Spiers and Maguire (2007) suggest that the hippocampus is involved when detailed information is necessary in performing the navigational task. Vividness of the memory or the amount of episodic details retrieved affects the level of activity in the hippocampus as well (Gilboa et al., 2004; Addis et al., 2007). This notion is also suggested by Moscovitch et al. (2006) in their review about the multiple trace theory and by Viskontas et al. (2009) based on results obtained in a prospective study in which decreased retrieval of episodic details resulted in decreased hippocampal activity (but see Wang et al., 2009). Here, we were able to align the memory strength of one contextual detail between recent and remote retrieval. Nevertheless, it is perceivable that the two conditions still differed in the amount of other, taskirrelevant details retrieved. Our paradigm does not provide evidence for such a difference in task-irrelevant retrieval. Yet, the idea that system-level consolidation involves the integration of new memories into existing ones by reducing irrelevant and overlapping details, embraces the possible retrieval differences in task-irrelevant contextual detail, a notion that might be probed by explicit studies in the future.

Can we observe consolidation effect at $24 \mathrm{~h}$ delay? According to lesion studies (Scoville and Milner, 1957; Zola-Morgan and Squire, 1990; Bontempi et al., 1999), this appears short when considering how long it takes for a memory trace to become hippocampally independent. However, changes are already present at the molecular level a few hours after the encoding in which synthesis of new proteins enables a memory trace to stabilize (Kandel, 2001). At the behavioral level, many prospective studies have shown consolidation effect after a night of sleep (for review, see Stickgold and Walker, 2007) or even a short nap (Mednick et al., 2003; Backhaus and Junghanns, 2006). In the imaging domain, there are also studies showing decreased hippocampal activity after $1 \mathrm{~d}$ (Bosshardt et al., 2005b; Takashima et al., 2006). Moreover, it has recently been shown that consolidation processes are accelerated if new information can be incorporated into a preexisting knowledge (Tse et al., 2007). Since subjects performed a training session with stimuli not used in the actual experiment $\sim 1$ week beforehand, and they performed a face item familiarization task before the actual memorization of the association to minimize effects of item memory, preexisting knowledge might have increased the speed of consolidation.

In addition to the memory representational areas, we also found activity increase in the prefrontal cortex with consolidation which is in line with previous studies (Rugg et al., 2002; Takashima et al., 2006; Gais et al., 2007; Sterpenich et al., 2007). The most prominent increase in our study was found in the inferior frontal gyrus bilaterally, including the Brodmann's areas 44 and 45. These areas are known to be involved in semantic processing (Cabeza and Nyberg, 2000; Thompson-Schill, 2003). Because of the nature of our task, it would have been beneficial to make use of a semantic strategy and to give meanings to the arbitrary associations for successful encoding and thus, differential activation in these areas might indicate that consolidation led to integration of memories into existing semantic knowledge. We did not find significant activity that was stronger for remote hits than recent hits in the ventromedial prefrontal cortex as reported previously (Frankland and Bontempi, 2005; Takashima et al., 2006; Gais et al., 2007). We are careful in interpreting this null finding, but possible reasons might include the relative short delay between remote and recent encoding and potentially reduced task-irrelevant recollection, because medial prefrontal effects occurred after longer delays only (Frankland and Bontempi, 2005; Takashima et al., 2006; Gais et al., 2007) and it has been associated with recollection (reviews by Svoboda et al., 2006; Cabeza and St Jacques, 2007).

It is interesting to point out that both remote and recent hits showed connectivity with early visual areas. These areas were more strongly connected with the posterior hippocampus for the recent condition and with the FFA for the remote condition. The engagement of the early visual areas might reflect a process in which the visual features of the memory traces are constructed (Slotnick, 2004). In a magnetoencephalographic study using the same stimuli as here, Nieuwenhuis et al. (2008) found oscillatory gamma band activity in early visual areas that was associated with memory retrieval, i.e., suggestive of a constructive process as well. We posit that the hippocampus engages visual areas for unconsolidated memory to construct the locations of the spatial cue. With consolidation, this construction process might directly be initiated by the FFA.

In conclusion, we report a network change in face-location association retrieval after a delay of $24 \mathrm{~h}$, a shift from a hippocampus dependent network to a neocortical one. While consolidation is thought of as memory stabilization, it is not a mere strengthening of the initial memory trace. With time, the memory undergoes changes that putatively include generalization, degradation, simplification, interference, and integration into existing representations.

\section{References}

Addis DR, McAndrews MP (2006) Prefrontal and hippocampal contributions to the generation and binding of semantic associations during successful encoding. Neuroimage 33:1194-1206.

Addis DR, Moscovitch M, Crawley AP, McAndrews MP (2004) Recollective qualities modulate hippocampal activation during autobiographical memory retrieval. Hippocampus 14:752-762.

Addis DR, Moscovitch M, McAndrews MP (2007) Consequences of hippocampal damage across the autobiographical memory network in left temporal lobe epilepsy. Brain 130:2327-2342.

Alvarez P, Squire LR (1994) Memory consolidation and the medial temporal lobe: a simple network model. Proc Natl Acad Sci U S A 91:7041-7045.

Ashburner J, Friston KJ (2005) Unified segmentation. Neuroimage 26:839-851.

Backhaus J, Junghanns K (2006) Daytime naps improve procedural motor memory. Sleep Med 7:508-512.

Bontempi B, Laurent-Demir C, Destrade C, Jaffard R (1999) Timedependent reorganization of brain circuitry underlying long-term memory storage. Nature 400:671-675.

Bosshardt S, Degonda N, Schmidt CF, Boesiger P, Nitsch RM, Hock C, Henke K (2005a) One month of human memory consolidation enhances retrieval-related hippocampal activity. Hippocampus 15:1026-1040.

Bosshardt S, Schmidt CF, Jaermann T, Degonda N, Boesiger P, Nitsch RM, Hock C, Henke K (2005b) Effects of memory consolidation on human hippocampal activity during retrieval. Cortex 41:486-498.

Cabeza R, Nyberg L (2000) Imaging cognition II: an empirical review of 275 PET and fMRI studies. J Cogn Neurosci 12:1-47.

Cabeza R, St Jacques P (2007) Functional neuroimaging of autobiographical memory. Trends Cogn Sci 11:219-227.

Diana RA, Yonelinas AP, Ranganath C (2007) Imaging recollection and familiarity in the medial temporal lobe: a three-component model. Trends Cogn Sci 11:379-386.

Frankland PW, Bontempi B (2005) The organization of recent and remote memories. Nat Rev Neurosci 6:119-130. 
Friston KJ, Buechel C, Fink GR, Morris J, Rolls E, Dolan RJ (1997) Psychophysiological and modulatory interactions in neuroimaging. Neuroimage 6:218-229.

Gais S, Albouy G, Boly M, Dang-Vu TT, Darsaud A, Desseilles M, Rauchs G, Schabus M, Sterpenich V, Vandewalle G, Maquet P, Peigneux P (2007) Sleep transforms the cerebral trace of declarative memories. Proc Natl Acad Sci U S A 104:18778-18783.

Gilboa A, Winocur G, Grady CL, Hevenor SJ, Moscovitch M (2004) Remembering our past: functional neuroanatomy of recollection of recent and very remote personal events. Cereb Cortex 14:1214-1225.

Grunwald T, Lehnertz K, Heinze HJ, Helmstaedter C, Elger CE (1998) Verbal novelty detection within the human hippocampus proper. Proc Natl Acad Sci U S A 95:3193-3197.

Hayasaka S, Nichols TE (2003) Validating cluster size inference: random field and permutation methods. Neuroimage 20:2343-2356.

Hirabayashi T, Miyashita Y (2005) Dynamically modulated spike correlation in monkey inferior temporal cortex depending on the feature configuration within a whole object. J Neurosci 25:10299-10307.

Janzen G, Jansen C, van Turennout M (2008) Memory consolidation of landmarks in good navigators. Hippocampus 18:40-47.

Kandel ER (2001) The molecular biology of memory storage: a dialogue between genes and synapses. Science 294:1030-1038.

Kanwisher N, Yovel G (2006) The fusiform face area: a cortical region specialized for the perception of faces. Philos Trans R Soc Lond B Biol Sci 361:2109-2128.

Levine B, Turner GR, Tisserand D, Hevenor SJ, Graham SJ, McIntosh AR (2004) The functional neuroanatomy of episodic and semantic autobiographical remembering: a prospective functional MRI study. J Cogn Neurosci 16:1633-1646.

Levine DN, Warach J, Farah M (1985) Two visual systems in mental imagery: dissociation of "what" and "where" in imagery disorders due to bilateral posterior cerebral lesions. Neurology 35:1010-1018.

Marr D (1970) A theory for cerebral neocortex. Proc R Soc Lond B Biol Sci 176:161-234.

Marr D (1971) Simple memory: a theory for archicortex. Philos Trans R Soc Lond B Biol Sci 262:23-81.

Mayes A, Montaldi D, Migo E (2007) Associative memory and the medial temporal lobes. Trends Cogn Sci 11:126-135.

McClelland JL, McNaughton BL, O'Reilly RC (1995) Why there are complementary learning systems in the hippocampus and neocortex: insights from the successes and failures of connectionist models of learning and memory. Psychol Rev 102:419-457.

Mednick S, Nakayama K, Stickgold R (2003) Sleep-dependent learning: a nap is as good as a night. Nat Neurosci 6:697-698.

Moscovitch M, Nadel L, Winocur G, Gilboa A, Rosenbaum RS (2006) The cognitive neuroscience of remote episodic, semantic and spatial memory. Curr Opin Neurobiol 16:179-190.

Muñoz M, Insausti R (2005) Cortical efferents of the entorhinal cortex and the adjacent parahippocampal region in the monkey (Macaca fascicularis). Eur J Neurosci 22:1368-1388.

Nadel L, Moscovitch M (1997) Memory consolidation, retrograde amnesia and the hippocampal complex. Curr Opin Neurobiol 7:217-227.

Nieuwenhuis IL, Takashima A, Oostenveld R, Fernández G, Jensen O (2008) Visual areas become less engaged in associative recall following memory stabilization. Neuroimage 40:1319-1327.

O’Reilly RC, Norman KA (2002) Hippocampal and neocortical contributions to memory: advances in the complementary learning systems framework. Trends Cogn Sci 6:505-510.

Paz R, Bauer EP, Paré D (2007) Learning-related facilitation of rhinal interactions by medial prefrontal inputs. J Neurosci 27:6542-6551.

Penny WD, Holmes AP, Friston KJ (2003) Random effects analysis. In: Hu- man brain function, Ed 2 (Frackowiak RSJ, Friston KJ, Frith C, Dolan R, Price CJ, Zeki S, Ashburner J, Penny WD, eds). San Diego: Academic.

Piefke M, Fink GR (2005) Recollections of one's own past: the effects of aging and gender on the neural mechanisms of episodic autobiographical memory. Anat Embryol (Berl) 210:497-512.

Rekkas PV, Constable RT (2005) Evidence that autobiographic memory retrieval does not become independent of the hippocampus: an fMRI study contrasting very recent with remote events. J Cogn Neurosci 17:1950-1961.

Rugg MD, Otten LJ, Henson RN (2002) The neural basis of episodic memory: evidence from functional neuroimaging. Philos Trans R Soc Lond B Biol Sci 357:1097-1110.

Scoville WB, Milner B (1957) Loss of recent memory after bilateral hippocampal lesions. J Neurol Neurosurg Psychiatry 20:11-21.

Slotnick SD (2004) Visual memory and visual perception recruit common neural substrates. Behav Cogn Neurosci Rev 3:207-221.

Smith CN, Squire LR (2009) Medial temporal lobe activity during retrieval of semantic memory is related to the age of the memory. J Neurosci 29:930-938.

Spiers HJ, Maguire EA (2007) The neuroscience of remote spatial memory: a tale of two cities. Neuroscience 149:7-27.

Squire LR, Zola-Morgan S (1991) The medial temporal lobe memory system. Science 253:1380-1386.

Sterpenich V, Albouy G, Boly M, Vandewalle G, Darsaud A, Balteau E, Dang-Vu TT, Desseilles M, D’Argembeau A, Gais S, Rauchs G, Schabus M, Degueldre C, Luxen A, Collette F, Maquet P (2007) Sleep-related hippocampo-cortical interplay during emotional memory recollection. PLoS Biol 5:e282.

Stickgold R, Walker MP (2007) Sleep-dependent memory consolidation and reconsolidation. Sleep Med 8:331-343.

Suzuki WA, Eichenbaum H (2000) The neurophysiology of memory. Ann N Y Acad Sci 911:175-191.

Svoboda E, McKinnon MC, Levine B (2006) The functional neuroanatomy of autobiographical memory: a meta-analysis. Neuropsychologia 44:2189-2208.

Takashima A, Petersson KM, Rutters F, Tendolkar I, Jensen O, Zwarts MJ, McNaughton BL, Fernández G (2006) Declarative memory consolidation in humans: a prospective functional magnetic resonance imaging study. Proc Natl Acad Sci U S A 103:756-761.

Takashima A, Nieuwenhuis IL, Rijpkema M, Petersson KM, Jensen O, Fernández G (2007) Memory trace stabilization leads to large-scale changes in the retrieval network: a functional MRI study on associative memory. Learn Mem 14:472-479.

Takehara-Nishiuchi K, McNaughton BL (2008) Spontaneous changes of neocortical code for associative memory during consolidation. Science 322:960-963.

Thompson-Schill SL (2003) Neuroimaging studies of semantic memory: inferring "how" from "where." Neuropsychologia 41:280-292.

Tse D, Langston RF, Kakeyama M, Bethus I, Spooner PA, Wood ER, Witter MP, Morris RG (2007) Schemas and memory consolidation. Science 316:76-82.

Verhagen L, Grol MJ, Dijkerman HC, Toni I (2006) Studying visuallyguided reach to grasp movements in an MR-environment. Neuroimage 31:S45.

Viskontas IV, Carr VA, Engel SA, Knowlton BJ (2009) The neural correlates of recollection: hippocampal activation declines as episodic memory fades. Hippocampus 19:265-272.

Wang SH, Teixeira CM, Wheeler AL, Frankland PW (2009) The precision of remote context memories does not require the hippocampus. Nat Neurosci 12:253-255.

Zola-Morgan SM, Squire LR (1990) The primate hippocampal formation: evidence for a time-limited role in memory storage. Science 250:288-290. 